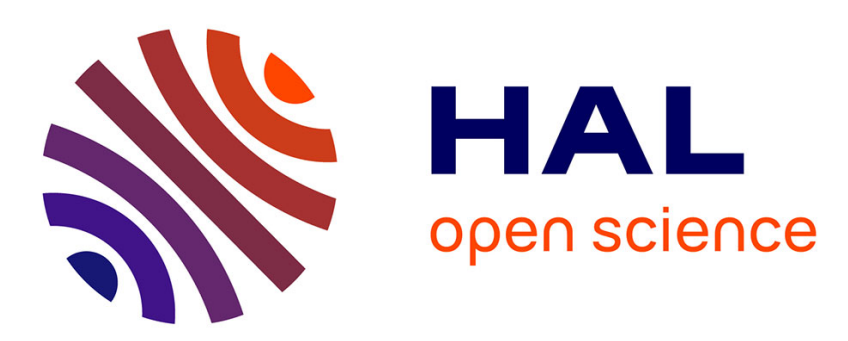

\title{
Importance of coil-overlapping for the effectiveness of hydroxypropylguars as water retention agent in cement-based mortars
}

\author{
Thomas Poinot, Alexandre Govin, Philippe Grosseau
}

\section{To cite this version:}

Thomas Poinot, Alexandre Govin, Philippe Grosseau. Importance of coil-overlapping for the effectiveness of hydroxypropylguars as water retention agent in cement-based mortars. Cement and Concrete Research, 2014, 56, pp.61-68. 10.1016/j.cemconres.2013.11.005 . hal-00936254

\section{HAL Id: hal-00936254 https://hal.science/hal-00936254}

Submitted on 27 Jan 2014

HAL is a multi-disciplinary open access archive for the deposit and dissemination of scientific research documents, whether they are published or not. The documents may come from teaching and research institutions in France or abroad, or from public or private research centers.
L'archive ouverte pluridisciplinaire HAL, est destinée au dépôt et à la diffusion de documents scientifiques de niveau recherche, publiés ou non, émanant des établissements d'enseignement et de recherche français ou étrangers, des laboratoires publics ou privés. 


\section{Importance of coil-overlapping}

\section{for the effectiveness of hydroxypropylguars}

\section{as water retention agent in cement-based mortars}

Thomas Poinot $^{\text {a }}$-Alexandre Govin ${ }^{a^{*}}$ — Philippe Grosseau ${ }^{\text {a }}$

${ }^{a}$ Ecole Nationale Supérieure des Mines, SPIN-EMSE, Département PROPICE,

CNRS:UMR5307, LGF, F-42023 Saint-Etienne

* Corresponding author: Tel: +33477420253

E-mail address: govin@emse.fr 


\section{ABSTRACT}

This paper is devoted to the role of hydroxypropylguars (HPG) on water retention (WR) capacities of cement-based mortars. WR and pore solution viscosity of mortars formulated with different dosage and kind of HPG were investigated. The results allowed us to rule-out that WR is only governed by pore solution viscosity. A sharp change in slope was observed on the curve WR vs. polymer dosage. This was attributed to the threshold dosage corresponding to the network formation of hydrocolloidal associated HPG molecules in pore solution. Below this dosage, no effect of HPG on WR was obtained. Above this dosage, HPG aggregates may stop the water flow by plugging the porous network of a thin HPG-enriched filter cake at the interface mortar-substrate. This study showed moreover that additional substitution of alkyl chain on HPG improves its effectiveness at low dosage. This was attributed to an enhancement of overlapping.

\section{KEYWORDS:}

polysaccharide $(D)$, hydroxypropylguar $(D)$, admixture $(D)$, mortar $(E)$, water retention $(C)$ 


\section{Introduction}

Hydroxypropylguars (HPG) are etherified derivatives of guar gum, a natural polysaccharide extracted from the seed endosperm of native plant from India Cyamopsis tetragonolobus. Due to their thickening effect, good solubility and thermal stability in solution, HPG are used in numerous industries such as textile printing, oil production or paper manufacturing [1]. HPG are also used in the field of building construction since they are introduced into mortar formulation as viscosity-enhancing and water retention admixtures.

Water retention (WR) is one of the key properties of mortar at fresh state. Indeed, water retention agent allows enhancing cement hydration by limiting the absorption of mixing water by the substrate. Thus, thanks to the WR agent, the mortar exhibits better mechanical and adhesive properties when it is applied in thin layer or on highly absorbent substrate [2,3]. Cellulose ethers (CE) are the most widely used WR agents. Therefore, up to now, research into the understanding of water retention in cement-based material is mainly focused on the effect of cellulose ethers. It appears that polymer molecular parameters, such as nature and content of substitution groups, and molecular weight, have a significant influence on WR $[4,5]$.

Desbrière studied in the mid-90s the influence of cellulose derivatives to the limitation of the fluid loss in cement slurries during static filtration under strong differential pressure (70 bars) [6]. The author proposed two predominant mechanisms: the increase in the viscosity of the interstitial solution and the reduction of the permeability of the cement cake. He reached these assumptions considering that the fluid flow through a porous media constituted by filter cake can be described by Darcy's law. Thus the fluid loss volume, the opposite of WR, is a function of the viscosity of the filtrate and the cake permeability.

Other authors use also Darcy's law to describe the flow of water in a porous media under a pressure gradient due to capillary suction or depression. In case of good water retention 
capacity, they assume that the amount of extracted water is low and hence the permeability of the sample remains almost constant. Thus water-soluble polymers improve WR by increasing the viscosity of the pore solution whatever the polymer molecular parameters and dosage [7,8]. In the same idea, many authors interpreted WR as the result of a reduction of the aqueous phase mobility due to an increase in viscosity when the polymer-to-cement ratio increases but without involving Darcy's law [9-11]. These authors explain also WR improvement in terms of sealing effect due to the formation of polymer water-impervious films in the paste (i.e. polysaccharides act as diffusion barrier to the water) [9-11]. Thanks to pulsed field gradient and NMR imaging investigations, Patural et al. [12] demonstrated that CE do not modify the water self-diffusion coefficient, even when an osmotic gradient is counteracting the diffusion flux. These results allow discarding the diffusion barrier hypothesis and showing that WR is governed by capillary suction. Then, by means of NMR dispersion investigations, Patural et al. [13] showed that the surface diffusion coefficient of water is not modify by the presence of CE, in spite of strong effect on solution's viscosity. However, CE increase the fraction of mobile water molecules transiently present at the solid surfaces. It was found a linear correlation between this fraction of mobile water and WR.

In the same time, by comparing the flow between a CE solution and a simple oil of similar viscosity from imbibitions experiments, Marlière et al. [14] have obtained different behaviors with these two solutions. Indeed, the flow stops much before with the CE solutions which demonstrate that WR is not only governed by the fluid viscosity.

The second hypothesis of Desbrière [6] to explain WR is the reduction of filter permeability. According to this author, this is the major parameter and it may be explained by (i) a decrease in the porosity due to adsorption of macromolecular chains on cement; (ii) a physical 
plugging of the pore; (iii) an increase in the cake compaction due to modification of the local structure.

The influence of CE on the mortar's permeability is difficult to determine since it is very difficult to measure it in a direct way at the fresh state and water transport properties of hardened mortars differs strongly from the fresh material [15-17]. Nevertheless, several studies discussed the impact of CE on the microstructure and porous structure of cementbased material in both fresh and hardened state $[3,4,18,19]$. It appears that a specific porous structure is generated by CE.

However, Marlière et al. [14] suggested that WR is not influenced by cement-CE interactions within a given timeframe. Indeed, they have shown that the mortar WR curves (WR vs CE dosage) exhibit similar shape when the cement is substituted by sand or glass beads. Bülichen et al. [20] demonstrated also that MHEC effectiveness as water retention agent is not linked to its adsorption on cement.

In accordance with Desbrière [6], the authors cited above considered that WR is explained by a reduction of filter cake permeability due to the formation of hydrocolloidal associated 3D polymer networks at high dosage (i.e. above a certain threshold concentration known as the overlapping concentration) which physically plug the pores of the paste [20-22]. Marlière et al. [14] described a jamming effect explained by the size and the quantity of polydisperse aggregates. This theory is consistent with the thin CE-enriched filter cake at the mortar/substrate interface suggested by Patural et al. [12], and with the accumulation of CE observed at the interface with the substrate in tile adhesive mortar [3,23] or in the uppermost millimeter of self-leveling flooring mortar layer [24].

This short state of the art highlights that the understanding of WR mechanism induced by CE is crucial and interests many authors. The importance of colloidal interactions has emerged recently while the increase in pore solution viscosity is still often advanced. However, few 
studies have given attention to the impact of HPG on mortar WR although they were presented by Plank in 2004 as promising new class of water retaining agent in building materials [25]. Indeed, in comparison with CE, HPG exhibit comparable water retention abilities [26-28], similar delayed effect on cement hydration [27,28] and a manufacturing process less polluting [29]. Furthermore, the HPG use is already well-established in industrial construction chemistry.

The aim of this work consists in understanding the effectiveness of HPG as water retention agent and comparing its behavior with classical CE. The main issue is to determine if WR is due to an increase in pore solution viscosity or to the formation of hydrocolloidal aggregates in the case of HPG. Thus working with non-cellulosic polysaccharides can also allow us to establish how generalizable are the recent findings concerning CE. For instance, other polysaccharidic admixtures such as starch $[5,26]$ and chitin derivatives [28] were found to act on WR in different ways.

The present work will be divided into two parts. The first one will consist in the study of the influence of pore solution viscosity on WR. The objective is to determine if a master curve appears whatever the admixture when WR is plotted against the pore solution viscosity. The second part will focus on the influence of the agglomerates formation due to coil-overlapping. To achieve this, the dosage and kind of HPG will be varied and the associate WR and viscosity of the extracted pore solution will be systematically characterized. Results will be compared to those obtained with classical cellulosic water retention agents. The influence of dosage on WR and the viscosity of admixed-mortars pore solutions will be first discussed. 


\section{Material and methods}

\subsection{Mineral and organic compounds}

\subsubsection{Mineral products}

The investigated ordinary Portland cement (OPC) was a CEM I 52.5 R CE CP2 NF type cement according to EN 197-1 and NF P 15-318 standards. Oxide composition was determined by X-ray fluorescence spectroscopy (SRS3400, Bruker-AXS). Phase composition was quantified by means of Rietveld refinement method (Siroquant V2.5 software) after XRD analysis (D5000, Siemens). Chemical and phase compositions of the cement used are given in Table 1.

Table 1: Chemical and phase compositions of the investigated cement.

\begin{tabular}{|cc|cc|cc|cc|}
\hline \multicolumn{4}{|c|}{ Chemical composition (\% wt) } & \multicolumn{4}{c|}{ Phase composition (\% wt) } \\
\hline Oxides & XRF & Oxides & XRF & Phases & $\begin{array}{c}\text { XRD } \\
\text { (Rietveld) }\end{array}$ & Phases & $\begin{array}{c}\text { XRD } \\
\text { (Rietveld) }\end{array}$ \\
\hline $\mathrm{CaO}$ & $66.9 \pm 0.8$ & $\mathrm{MgO}$ & $1.16 \pm 0.01$ & $\mathrm{C}_{3} \mathrm{~S}$ & $79.4 \pm 0.5$ & Gypsum & $1.3 \pm 0.2$ \\
$\mathrm{SiO}_{2}$ & $20.9 \pm 0.2$ & $\mathrm{TiO}_{2}$ & $0.32 \pm 0.03$ & $\mathrm{C}_{2} \mathrm{~S}$ & $8.2 \pm 0.4$ & Anhydrite & $3.2 \pm 0.2$ \\
$\mathrm{Al}_{2} \mathrm{O}_{3}$ & $4.7 \pm 0.1$ & $\mathrm{P}_{2} \mathrm{O}_{5}$ & $0.14 \pm 0.01$ & $\mathrm{C}_{3} \mathrm{~A}$ & $3.3 \pm 0.2$ & Hemi-hydrate & $0.8 \pm 0.3$ \\
$\mathrm{SO}_{3}$ & $2.4 \pm 0.2$ & $\mathrm{MnO}$ & $0.04 \pm 0.01$ & $\mathrm{C}_{4} \mathrm{AF}$ & $4.1 \pm 0.9$ & Free CaO & $0.5 \pm 0.2$ \\
$\mathrm{Fe}_{2} \mathrm{O}_{3}$ & $2.6 \pm 0.1$ & $\mathrm{~K}_{2} \mathrm{O}$ & $0.10 \pm 0.01$ & & & & \\
$\mathrm{LOI}$ & $2.1 \pm 0.1$ & & & & & & \\
\hline
\end{tabular}

\subsubsection{Organic admixtures}

Seven polysaccharidic water retention admixtures were selected for this study: two hydroxypropyl methyl cellulose (HPMC1 and HMPC 2) and five hydroxypropylguars (HPG 1, HPG 2, HPG 3, HPG 5 and HPG 6).

Guar gum is a galactomannan consisting of a $\beta(1-4)$-linked D-mannopyranose backbone, with random branchpoints of galactose via an $\alpha(1-6)$ linkage [30]. The ratio of mannose to 
galactose is about 1.8. HPG are obtained from the native guar gum via an irreversible nucleophilic substitution, using propylene oxide in the presence of an alkaline catalyst. As guar gum exhibits a high chemical reactivity and is soluble in cold water, the chemical modification of the native guar gum does not require hard reaction conditions of temperature and pressure, does not generate large quantity of by-products and needs weak purification procedure [29].

In order to compare HPG behavior with classical polysaccharidic admixtures, two cellulose derivatives were also tested. Cellulose is a polysaccharide composed of individual anhydroglucose units linked through $\beta(1-4)$ glycosidic bonds. Because of strong intermolecular hydrogen bridging bonds, cellulose is not soluble in water. Thereby, cellulose ethers are obtained by pretreating cellulose with base (alkali cellulose) in order to break hydrogen bonds and to make accessible the active groups for etherifying agents. In the case of HPMC, the substitution of the hydroxyl groups takes place by reacting alkali cellulose with the combination of two etherifying agent: propylene oxide and methylchloride.

The schematic structure of both molecules is shown in Fig. 1 (substituent positions are arbitrary). It appears that HPMC are linear polymers whereas HPG exhibit branched-chain structure. Table 2 presents a qualitative description of the admixtures used. The qualitative substitution degrees are provided by the manufacturers. The molar substitution ratio $\left(\mathrm{MS}_{\mathrm{HP}}\right)$ is defined as the number of moles of hydroxypropyl groups per mole of anhydroglucose unit and is less than 3 for our molecules. The degree of substitution, noted $\mathrm{DS}_{\mathrm{M}}$, represents the amount of methoxyl groups per anhydroglucose unit and is about 1.8 for HPMC 1 and HPMC 2 according to the manufacturer.

All the HPG samples, provided by Lamberti S.p.A, have similar molecular weight, about $2.10^{6}$ g.mol ${ }^{-1}$. The only difference between HPG 1, 2 and 3 is the molar substitution ratio. HPG 5 and 6 exhibit additional substitution (short or long alkyl chains). The two HPMC samples 
exhibit the same substitution degrees. The only difference between HPMC 1 and HPMC 2 is molecular weight: about $0.25 .10^{6}$ and $1.10^{6} \mathrm{~g} \cdot \mathrm{mol}^{-1}$ respectively.
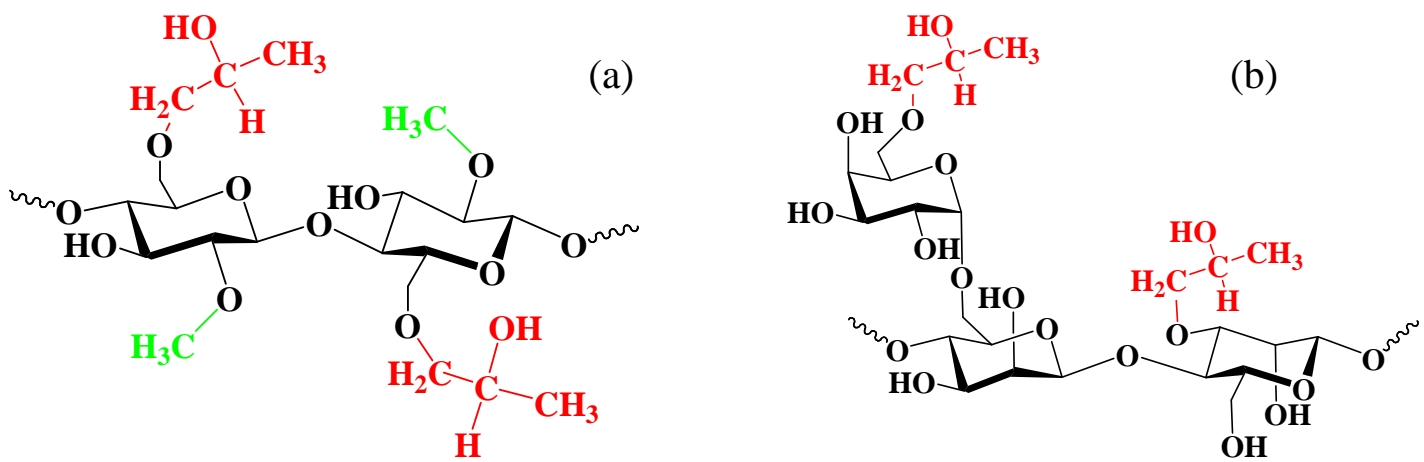

Fig. 1: Molecular structure of HPMC (a) and HPG (b).

Table 2: Qualitative description of the admixtures used.

\begin{tabular}{|c|cccc|}
\hline Admixtures & Viscosity & MS $_{\mathbf{H P}}$ & $\mathbf{D S}_{\mathbf{M}}$ & Additionnal substitution \\
\hline HPMC 1 & Very low & Very low & Very high & \\
HPMC 2 & Very high & Very low & Very high & \\
\hline HPG 1 & Medium & Low & $/$ & \\
HPG 2 & Medium & Medium & $/$ & \\
HPG 3 & Medium & High & $/$ & \\
HPG 5 & Medium & High & $/$ & Shorter alkyl chain \\
HPG 6 & Medium & High & $/$ & Longer alkyl chain \\
\hline
\end{tabular}

\subsection{Experimental methods of investigation}

\subsubsection{Preparation of mortars}

Admixtures were previously dissolved in deionized water by magnetic stirring for $24 \mathrm{~h}$ in order to obtain a complete dissolution of all polymers. The concentrations of these polymer solutions (from 1 to 16 g. $\mathrm{L}^{-1}$ ) allow obtaining, in mortars, polymer dosages varying from 0.1 to $1.6 \%$ by weight of cement (bwoc).

Then, mortars were prepared according to the following mixture proportions: $30 \mathrm{wt} . \%$ of cement, $65 \mathrm{wt} . \%$ of siliceous sand and $5 \mathrm{wt} . \%$ of limestone filler. The dry mixture (i.e. 
cement, sand and filler) was homogenized in a shaker (Turbula, Wab) for 10 min. Admixture solution was added in order to obtain a water to cement ratio $\mathrm{W} / \mathrm{C}=1$. Mortar mixing procedure was in accordance with EN 196-1 standard [31]. All tests were carried out at controlled temperature of $23^{\circ} \mathrm{C}$ because mortar fresh properties are temperature-dependent. A control test was performed with a mortar without admixture.

\subsubsection{Water retention measurements}

The water retention capacity of freshly-mixed mortar can be determine by means of different tests. Most of them are based on the measurement of the removed water after suction or depression. ASTM C1506-09 standard [32] describes one of these tests. ASTM measurements were performed 15 min after mixing in order to measure the water loss of mortar under depression. The standardized apparatus was submitted to a vacuum of $50 \mathrm{~mm}$ of mercury for 15 min. Then, water retention capacity, noted WR, was calculated using the following equation:

$W R(\%)=\frac{W_{0}-W_{1}}{W_{0}} \times 100$

$\mathrm{W}_{0}$ represents the initial mass of mixing water; $\mathrm{W}_{1}$ is the loss of water mass after aspiration.

\subsubsection{Viscosity of the pore solution}

The mortar pore solution was extracted from the mortar paste, by means of centrifugation, 15 min after mixing in order to be in the same conditions than WR test. A large volume of mortar (about $150 \mathrm{~mL}$ ) was centrifuged (Thermo Scientific, Sorvall Legend XF) for $10 \mathrm{~min}$ at 4,500 $\mathrm{rpm}$. The large volume allows having a representative sample. Then, the supernatant was collected and centrifuged again (Eppendrof, MiniSpin Plus) at higher speed (14,500 rpm) for $5 \mathrm{~min}$ in order to remove residual particles. Finally, rheological properties of the final supernatant is investigated by means of a rheometer (MCR 302, Anton-Paar) equipped with 
cone and plate geometry $\left(2^{\circ}\right.$ cone angle, $50 \mathrm{~mm}$ diameter $)$, thermostated at $20^{\circ} \mathrm{C}$. The steady shear flow of pore solutions have been investigated using decreasing logarithmic ramps in the $10^{3}-1 \mathrm{~s}^{-1}$ range.

Each mortar studied was divided into two parts after the mixing (section 2.2.1). One part allowed measuring WR (section 2.2.2) while the other one was centrifuged in order to determine pore solution viscosity (section 2.2.3). Each formulation (one polymer at one dosage) was prepared and studied twice.

\section{Results and discussions}

\subsection{Influence of polymer dosage on WR}

According to the NF DTU 26.1, mortars can be divided into three classes depending on their water retention capacities [33]. The first one (low retention) is for mortars that have water retention lower than $86 \%$. The second category (intermediate water retention) corresponds to values ranging from $86 \%$ to $94 \%$. The last one is defined by water retention higher than $94 \%$. This class is related to strong water retention mortars and is desired by formulators of industrial mortars.

Fig. 2 presents the water retention of mortars containing increasing dosages of admixture. The control test performed with a mortar without admixture exhibits a low WR value of $58.5 \pm 0.8$ $\%$. Then, as expected, WR is dosage dependent. The WR is improved as a result of increased amount of admixture, until reaching a plateau with very high WR values. The WR values are greater than $95 \%$ and therefore belong to the strong WR class. Moreover, about the shape of WR curves, an abrupt change in slope can be noticed. This issue will be discussed in more details further. 
Finally, it is worth mentioning that WR capability of HPG 5 is closed to HPMC 2 which is the best WR agent studied. With an enough dosage, around $0.6 \%$ bwoc, all the HPG allow achieving strong WR. On the contrary, HPMC 1 needs a very high dosage (about $1.4 \%$ bwoc) vto provide strong WR capacity to the mortar. These results confirm that HPG are good water retention agents, with efficiency comparable to CE.

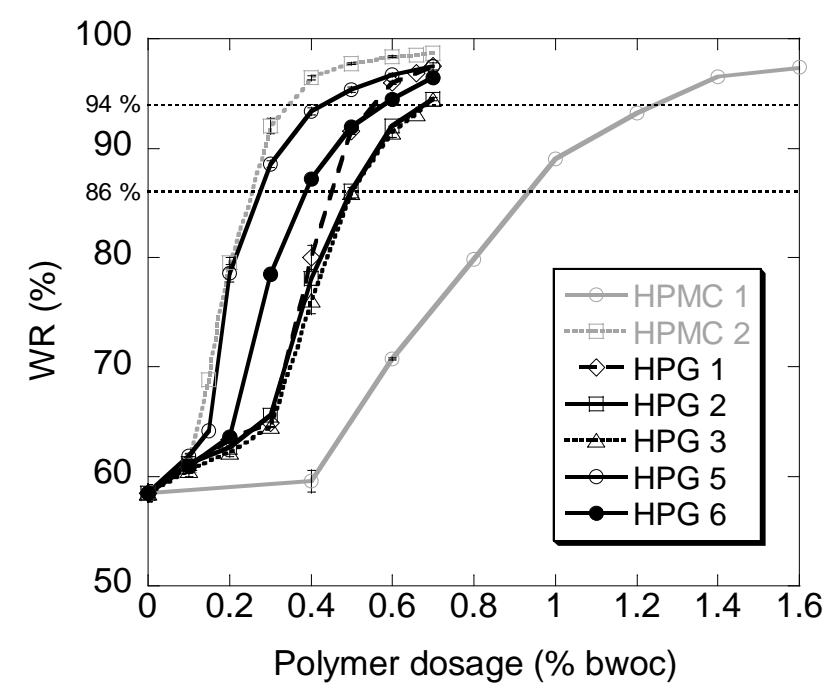

Fig. 2: Effect of polymer dosage on water retention of admixed mortars.

\subsection{Rheological properties of admixed-mortars pore solutions}

\subsubsection{Steady shear properties}

Typical flow curves of mortar pore solutions at different polymer dosages, obtained in case of HPG 5 admixed mortars, are shown in Fig. 3. In all cases, flow curves exhibited typical shear thinning behavior with a Newtonian region in the low shear rate range. The shear thinning nature of random coil polysaccharide solutions under flow is well known and due to entangled network systems $[34,35]$. At low shear rates, the disruption of entanglements by the imposed shear is balanced by the formation of new interactions, resulting in no net change in the extent of entanglements and hence no effect on the viscosity. This corresponds to the Newtonian plateau region, where the viscosity has a constant value called zero-shear rate apparent 
viscosity $\eta_{0}$. However, for higher shear rates, the rate of forced disentanglement predominates over the rate of entanglement of polymer chain. As a consequence, chains align in the flow direction and the viscosity decreases with the increasing shear rate.

The experimental data can be conveniently correlated with the Cross model:

$\eta(\dot{\gamma})=\eta_{\infty}+\frac{\eta_{0}-\eta_{\infty}}{1+(\tau \dot{\gamma})^{n}}$

where $\eta_{0}$ is the zero-shear rate viscosity, $\eta_{\infty}$ is the infinite rate viscosity, $\tau$ is a relaxation time and $\mathrm{n}$ a non-dimensional exponent.

Among Cross parameter, the zero-shear rate viscosity $\eta_{0}$ will be used in order to characterize the viscosity of the mortars pore solutions. Nevertheless, experimental data deviate from the model at low shear rate, especially for low dosage (Fig. 3). According to Clasen and Kulicke [36], the hydrophobic character of the side chains (hydropropyl for HPG, hydroxypropyl and methyl for HPMC) leads to strong intermolecular interactions and to the formation of 3D physical cross-linking (called superstructures) due to association. These associations determine the flow behavior at low shear rates and induce higher viscosity. When the shear rate increases, it becomes possible to overcome these hydrophobic interactions and thus to break the associated superstructures. This induces first a viscosity drop and then the classical flow behavior, with the Newtonian plateau at the zero-shear viscosity value and shear thinning at higher shear rates. Therefore the Cross model can be adjusted and the zero-shear viscosity can be determined by not taken account of data before the Newtonian regime. 


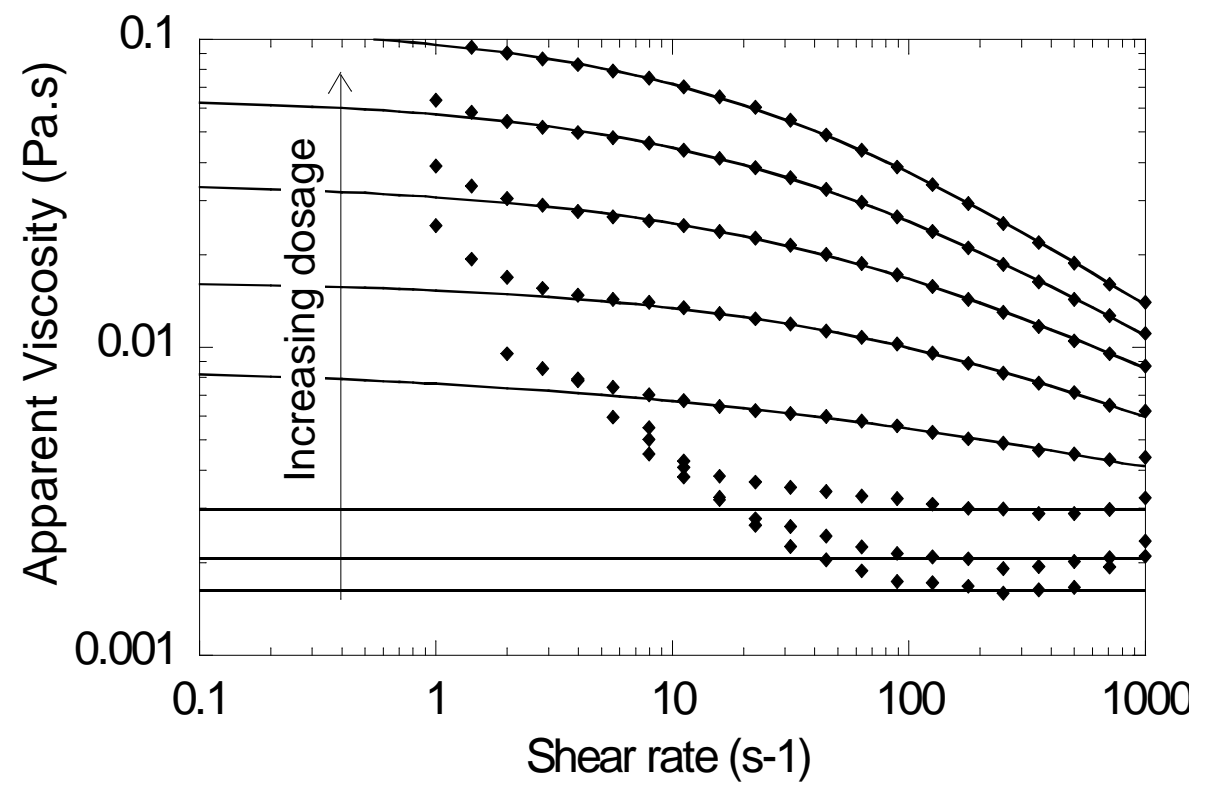

Fig. 3: Flow curves of mortars pore solutions for different polymer dosages - Example of HPG 5 - The full lines represent the calculated values according to the Cross model (Eq. 2).

\subsubsection{Concentration dependence of zero-shear viscosity}

As seen above, polymer concentration is an important parameter in controlling the rheological properties of polysaccharide solutions. At low polymer concentration, individual polymer molecules are present as isolated coils which provide their individual contribution to the viscosity. As the concentration of polymer is increased, the coils begin to bring into contact with one another. The corresponding concentration is called coil-overlap concentration (often noted $\left.\mathrm{C}^{*}\right)$. Above this concentration, a dramatic change in flow behavior occurs which results in a sudden increase in the concentration-dependence of zero-shear rate viscosity $[34,35,37]$. Therefore a double logarithmic plots of $\eta_{0}$ versus the concentration, as shown in Fig. 4 , clearly evidences the onset of significant coil overlap and interpenetration. Indeed, two welldefined linear dependencies can be observed. The dependence of $\eta_{0}$ on concentration is described by two power-law type correlations $\left(\eta_{0}=\mathrm{aC}^{\mathrm{b}}\right)$ where the exponent $\mathrm{b}$ differs depending on the regime considered. In our case, values of exponent obtained are consistent with the values found in literature [35,37-41]. Thus the polymer dosage corresponding to the 
intersection of the two straight lines for each admixture can be attributed to the boundary between semi-diluted and concentrated regime. This critical polymer dosage corresponds to the formation of polymer agglomerates in the pore solution. These data are collected in the Table 2.

It is worth noting that these critical dosages are not coil-overlap concentrations as defined in literature. Indeed, the real concentration of polymer present in pore solution is unknown for a given initial dosage, because (i) a certain amount of polymer is retained into the mortar paste or adsorbed onto cement phases and (ii) a non-negligible amount of water is lost due to cement hydration and mortar drying. The only known concentration is the introduced concentration, given in percentage by weight of cement (called polymer dosage) or in grams per liter (called introduced concentration) in the following.

Since $\eta_{0}$ was chosen to characterize the viscosity of mortars pore solutions, Fig. 4 allows also comparing the viscosifying properties of admixtures studied. It is obvious that HPMC 2 exhibits the stronger viscosity-enhancing effect, far ahead HPG, while HPMC 1 is the worst viscosifying agent. The difference observed between HPMC 1 and 2 is attributed to the difference in molecular mass.

Among HPG, pore solution viscosities of mortars admixed with HPG 1, 2 and 3 are similar. The only difference between these HPG is the substitution degree. In literature, it is indicated that $\eta_{0}$ decreases when DS increases [42]. The same effect was observed when polymers were dissolved in water. However, in mortars, increasing substitution degree leads to a decrease in polymer adsorption on cement phases $[27,43]$ and hence an increase in polymer amount in pore solution. Finally, the effect of substitution degree on viscosity is compensated by the effect of adsorption ability. Thus pore solutions have comparable viscosities. Therefore the formation of polymer aggregates occurs at similar polymer dosage of about $0.25 \%$ bwoc (which corresponds to an introduced concentration of 2.5 g. $\mathrm{L}^{-1}$ ) for HPG 1-2-3 (Table 2). 
On the contrary, the presence of additional alkyl chains (HPG 5-6) leads to the formation of polymer associates at slightly lower polymer dosage (Table 2). The interconnection between alkyl chains creates intermolecular interactions through specific hydrophobic interactions which cause a decrease in the coil-overlapping concentration [44].

The critical polymer dosage for HPMC 2 was found to be similar to HPG 5 ( $0.2 \%$ bwoc or 2 g. $\mathrm{L}^{-1}$ ) while the viscosifying effect is stronger. A relatively high dosage of almost $0.6 \%$ bwoc $\left(6 \mathrm{~g} . \mathrm{L}^{-1}\right)$ is necessary for the overlapping of HPMC 1 coils.

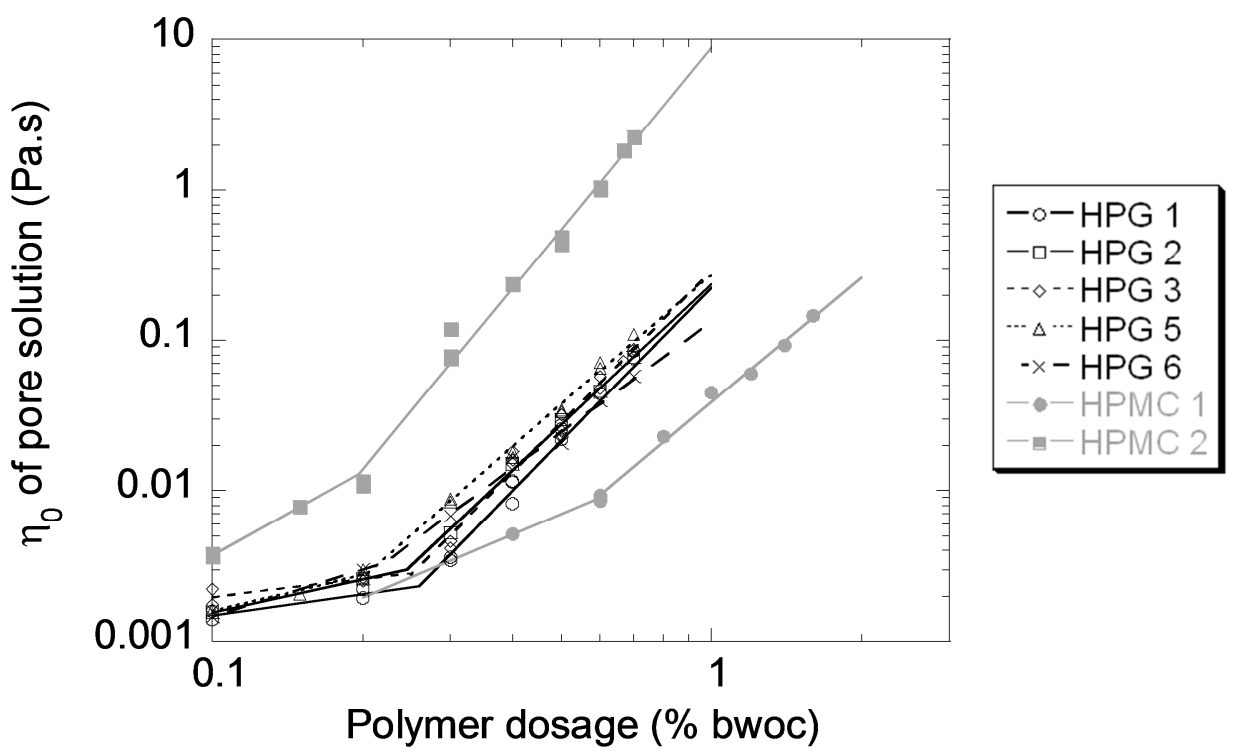

Fig. 4: Concentration dependence of zero-shear viscosity for all the admixtures studied

Table 2: Polymer dosage corresponding to the formation of polymer agglomerates in the mortar pore solution according to the admixture

\begin{tabular}{|c|c|c|c|c|c|c|c|}
\cline { 2 - 7 } \multicolumn{1}{c|}{} & HMPC1 & HPMC2 & HPG1 & HPG2 & HPG3 & HPG5 & HPG6 \\
\hline $\begin{array}{c}\text { Polymer dosage \% bwoc } \\
\text { (introduced concentration, g.L }\end{array}$ & 0.58 & 0.20 & 0.26 & 0.25 & 0.25 & 0.20 & 0.22 \\
$\begin{array}{c}\text { corresponding to formation } \\
\text { of agglomerates }\end{array}$ & $(5.8)$ & $(2.0)$ & $(2.6)$ & $(2.5)$ & $(2.5)$ & $(2.0)$ & $(2.2)$ \\
\hline
\end{tabular}




\subsection{Working mechanism of HPG as water retention agent}

The previous section highlights that an increase in polymer dosage leads to an increase in WR and in pore solution viscosity, and to the formation of agglomerates. The remaining question is to determine which parameter governs WR induced by HPG: viscosifying effect on pore solution or entanglement of HPG coils (i.e. formation of agglomerates)? However, the main difficulty was to discriminate these two factors, since the entanglement of HPG coils leads to a sharp increase in viscosity.

\subsubsection{Influence of pore solution viscosity}

In order to determine the impact of HPG thickening properties on WR, we have plotted WR versus the related pore solution viscosity, for each admixture studied (Fig. 5). It actually appears that WR increases with the pore solution viscosity (Fig. 5-a). From very low viscosities, near water (i.e. $\left.10^{-3} \mathrm{~Pa} . \mathrm{s}\right)$, viscosities up to a thousand times higher while WR begins at $60 \%$, equivalent to the control without admixture, and increases until reach the plateau near $100 \%$.

However, by zooming, it becomes clear that for a given pore solution viscosity, there are huge differences in WR, and a given WR could be reached with pore solutions of different viscosities. For instance, Fig. 5-b shows WR in the $0.001-0.01$ pore solution viscosity range. All data points are around WR values of $60-65 \%$ which corresponds to the control, except for HPG 1, HPG 6 and especially HPG 5. Indeed, with pore solution viscosity lower than 0.003 Pa.s, all admixtures induce low or no WR in comparison to the control, while HPG 5 induces WR close to $80 \%$. In the same way, when HPMC enhances pore solution viscosity up to 0.01 Pa.s, the related WR is only of 70\%. But for the same viscosity, HPG 1 and HPG 6 exhibit a WR of $80 \%$ and HPG 5 a WR of $87 \%$. On Fig. 5-c, where pore solution viscosity is ranging from 0.01 to 0.1 Pa.s, a strong difference in WR is observed for a given viscosity, from 5 to $18 \%$, whatever the admixture. Here again HPG 1, 5 and 6 allow obtaining the best WR. 
These figures show also that it is possible to obtain the same WR with pore solutions exhibiting different viscosities. In fact, viscosity can be multiplied by 5 while WR remains lower than $65 \%$ (Fig. 5-b). Mortars can exhibit WR of about $80 \%$ with pore solution viscosity varying strongly from 0.003 to 0.02 Pa.s (Fig. 5-a). For high WR ranging from 90 to $95 \%$, viscosities obtained vary in the range 0.015 - 0.090 Pa.s (Fig. 5-c). For the same WR (80\%), pore solution of mortars admixed with HPMC 2 and HPMC 1 exhibit viscosities of 0.011 and 0.023 Pa.s respectively (Fig. 5-c).

Finally, it is clear that pore solution viscosity cannot govern alone the WR of admixed mortars, as previously shown in the case of CE [14] and in agreement with the remark of Patural et al. [12] (i.e. some viscosifying admixtures such as starch, polyethylene oxides or polyacrylamides do not provide WR). 


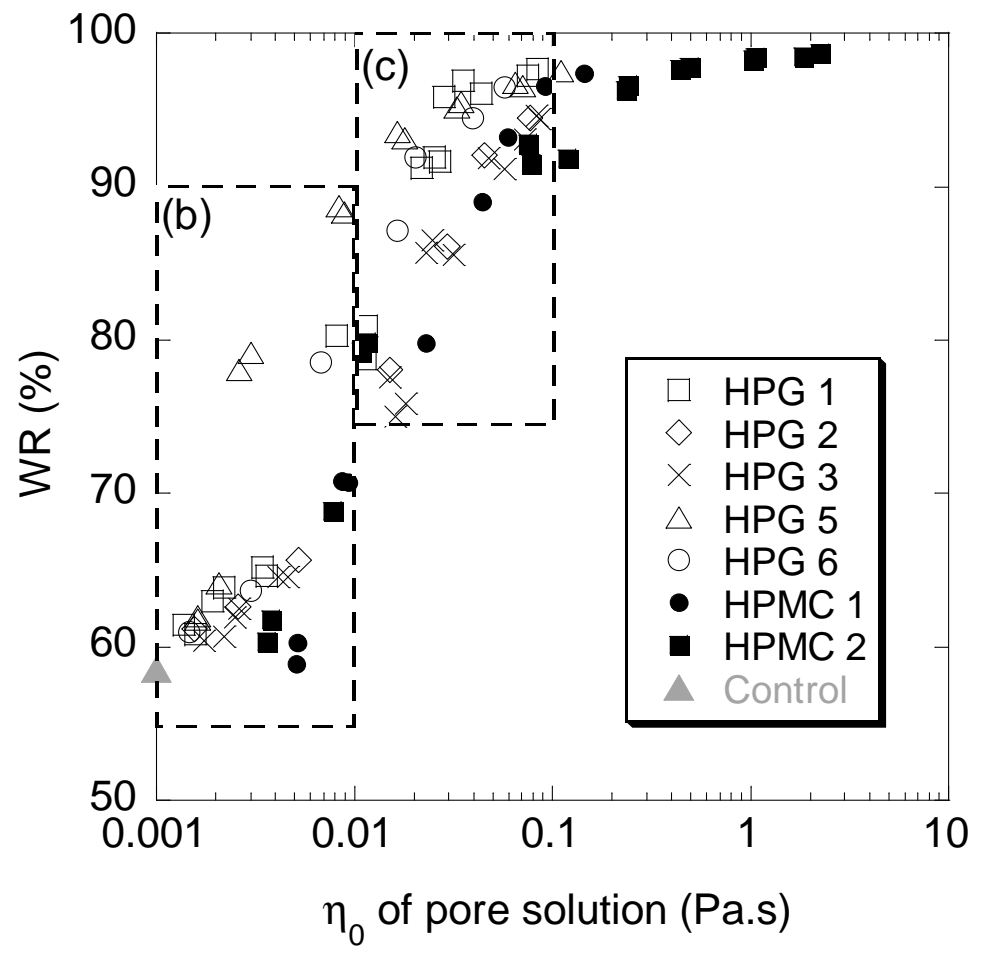

(a)
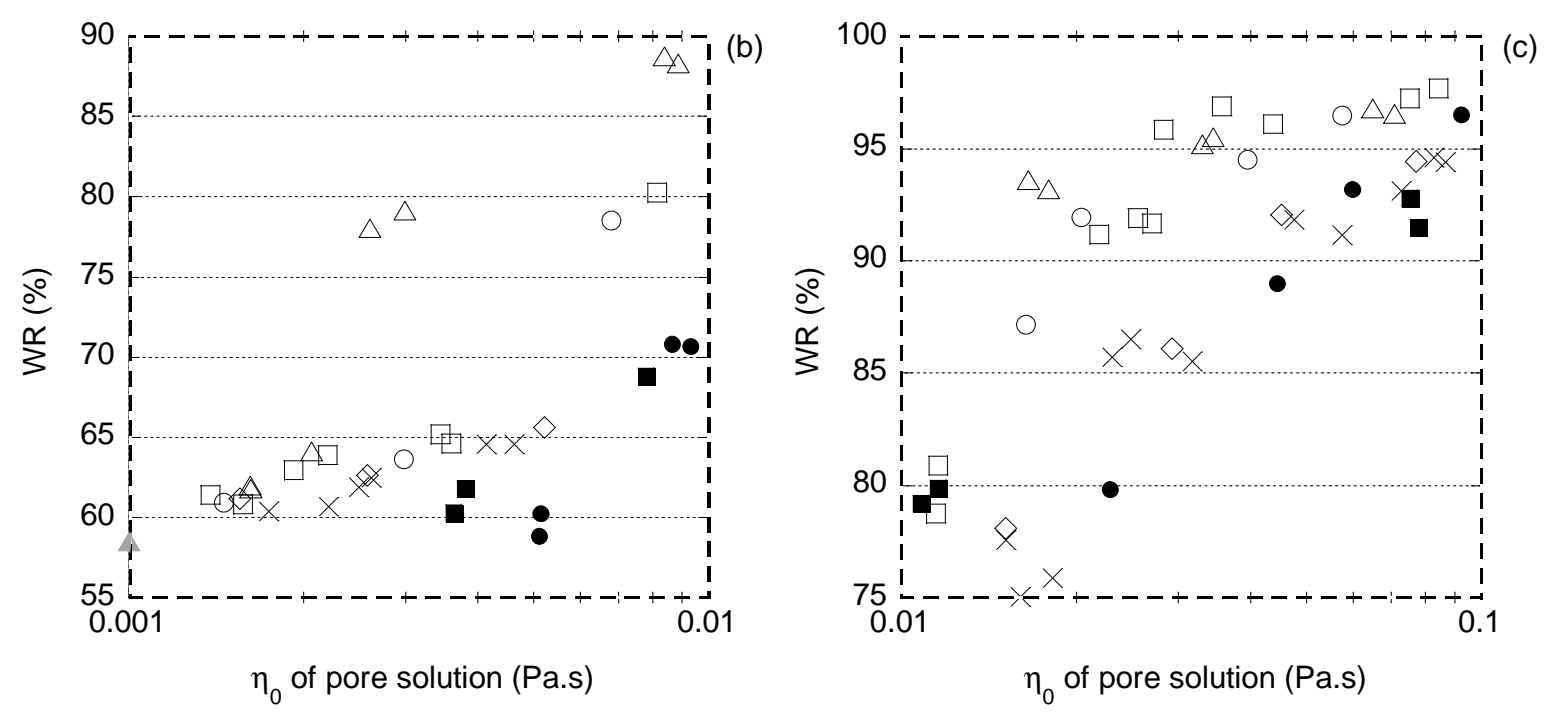

Fig. 5: Effect of pore solution viscosity on water retention from overall view (a), zoom in low viscosities (b) and zoom in middle viscosities (c)

\subsubsection{Influence of coil-overlapping on WR}

Fig. 6 represents both WR and pore solution viscosity (characterized by $\eta_{0}$ ) as a function of polymer dosage for each admixture. The impact of coil-overlapping on WR was highlighted by plotting also in dashed vertical line the dosage corresponding to the formation of polymer agglomerates (from Table 2). 
For the 5 HPG samples studied, it is obvious that the shape of WR curves is strongly influenced by the formation of polymer associates. Below this threshold dosage, no or low increase $(<3 \%)$ in WR is observed in comparison with the control test without admixture. Above the critical dosage, a sudden and sharp rise in WR is obtained.

Above the considered threshold dosage, the viscosity of the mortar pore solution exhibits also a strong and abrupt increase. Nevertheless, it has been shown above that the increase in pore solution viscosity cannot explain the WR induced by HPG. Therefore, based on these results and in agreement with literature considering CE [14,20], it appears clearly that the increase in WR in presence of HPG is due to the overlapping of HPG coils.

When the mortar is applied to absorbent substrates, few amount of water is lost first. This initial lost water flow leads to the formation of a thin HPG-enriched filter cake at the interface mortar-substrate $[3,12,23]$. At sufficient dosage, coil-overlapping occurs. HPG associates thus formed can physically plug the porous network and therefore stop the water flow. Once the water flow stopped, it can be considered from a macroscopic point of view that HPG provides WR.

This theory shows that the amount and/or size of polymer agglomerates are the key parameter for the WR mechanism, but the size of the filter cake porous network too. This assumption is consistent with the fact that water transport in fresh mortars is intrinsically related to the particle size distribution of the binder [17] and granular media [15,16].

The cases of HPG 5 and 6 are interesting. Indeed, for instance HPG 5 exhibits the best WR at a given dosage in comparison with other HPG, and slightly lower WR than HPMC 2 (Fig. 2). In addition, at a given induced viscosity of pore solution, HPG 5 and 6 are by far the best WR agents even in comparison with HPMC 2 (Fig. 5). Thus a positive impact of the substitution of alkyl chains can be suggested. In previous study [27], it has been assumed that the presence of the alkyl chains could cause steric hindrance which could reduce the adsorption ability. If 
the adsorption is reduced, more polymer molecules are actually in solution and hence effective to provide WR capacity. Nevertheless, the adsorption of non-ionic polysaccharide is a matter of controversy. According to Bülichen et al. [20], the considered adsorption could be actually a physical retention at high dosage. More likely, the alkyl chains enhance the entanglement of coils and thus increase the size and/or the amount of polymer aggregates at low dosage. This may explain the surprising high WR induced by HPG with additional alkyl chains, even with a weak effect on pore solution viscosity. This observation confirms that the relationship between pore solution viscosity and WR is not obvious.

By comparing three similar HPG which differ only in the degree of substitution (HPG 1, 2 and 3, see Table 2), it appears that HPG 1 exhibits better WR efficiency than HPG 2 and 3 for a given induced pore solution viscosity (without regarding the polymer dosage). Thus a low degree of substitution (HPG 1) leads to better WR for a similar effect on pore solution viscosity. During HPG manufacturing, hydroxyl groups are substituted by propylene oxide. The resulting hydroxypropyl groups replace strong intermolecular polar $\mathrm{OH}-\mathrm{OH}$ interactions with medium strength hydrophilic-hydrophobic bonds [45]. Indeed, the hydroxypropyl groups sterically block the hydrogen bondings sites on the guar backbone. Finally an increase in substitution degree induces a reduction of the entanglement between polysaccharide chains. Thus HPG 1 with low substitution degree exhibits stronger entanglement than HPG 2 and 3 at the same viscosity, and therefore a better WR.

The behavior of HPMC is more difficult to assess because of a lack of WR values below the threshold dosage. It appears nevertheless a significant WR effectiveness before the formation of the agglomerates (around $+10 \%$ for HPMC 2). According to Bülichen et al. [20,21], CE are able to achieve WR before the formation of hydrocolloidal associated polymer network by water intramolecular sorption and concomitant swelling. 


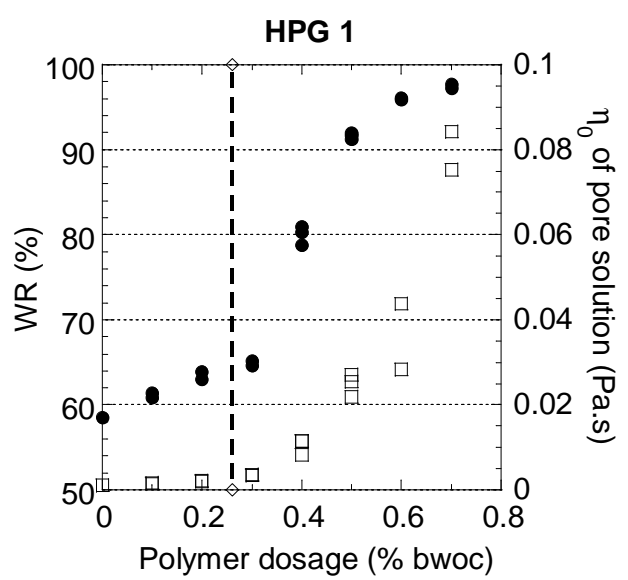

- WR

$\square \quad \eta_{0}$ of pore solution

- - Formation of polymer agglomerates
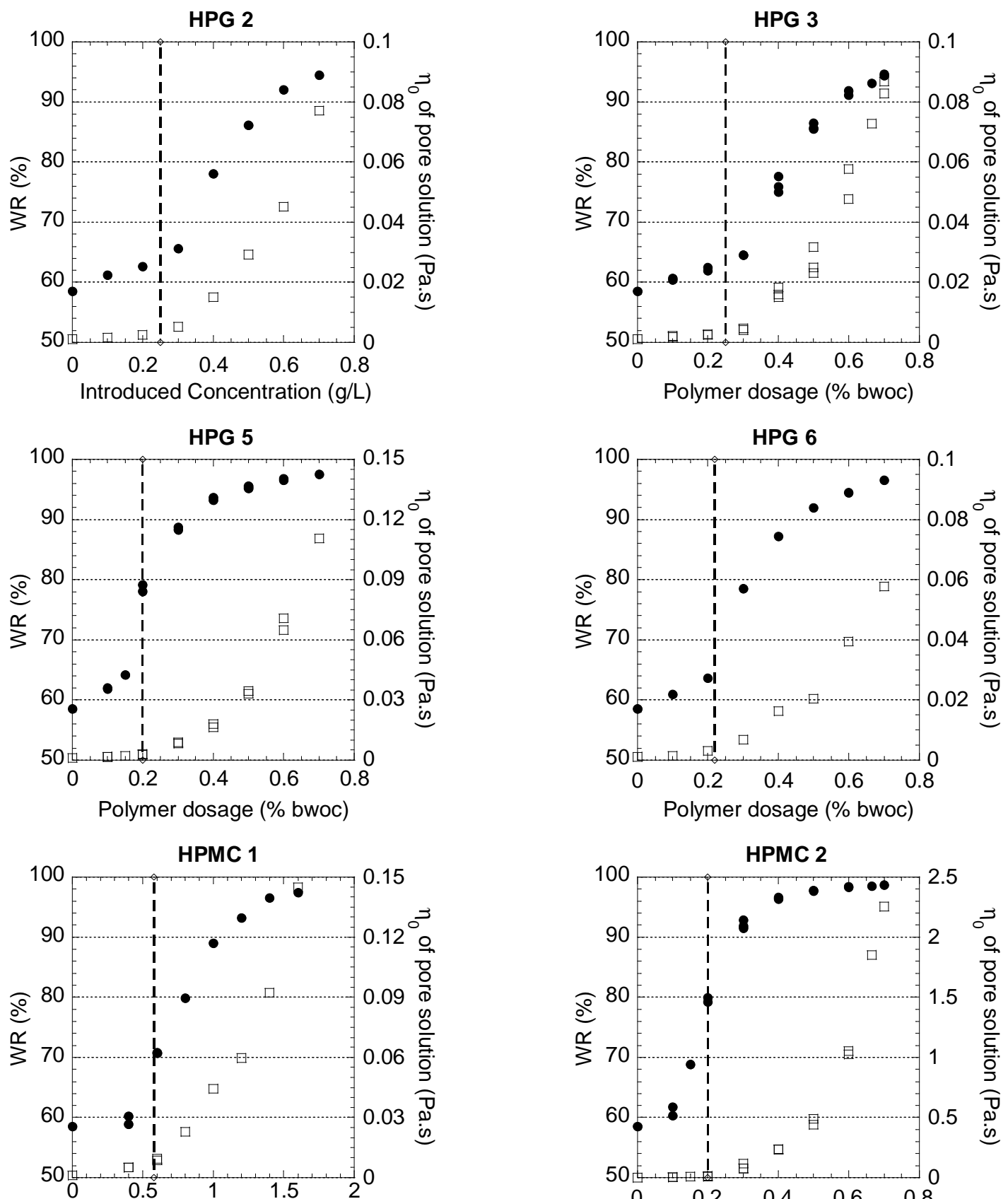

Polymer dosage (\% bwoc)

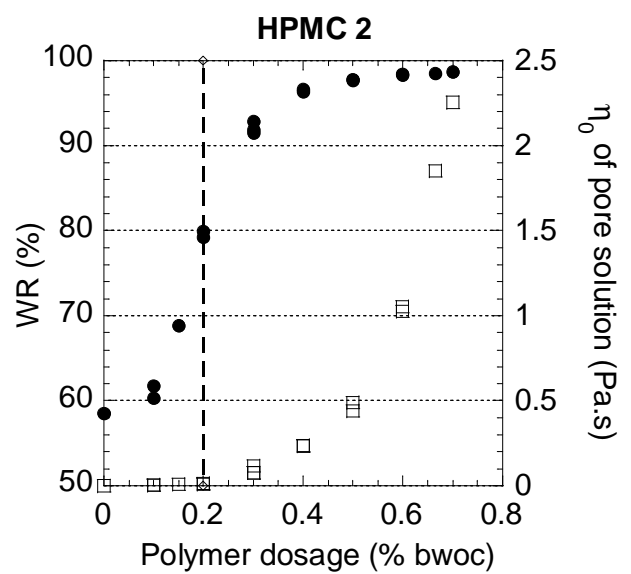

Fig. 6: Effect of polymer agglomerates formation on WR 


\section{Conclusions}

This study confirms that HPG provide strong WR to freshly-mixed mortars, in the same way than CE. As expected, WR is concentration dependant. Nevertheless, a sharp change in slope is observed on the WR curve of HPG-admixed mortars. This is attributed to the threshold dosage corresponding to the formation of hydrocolloidal associated HPG molecules network. This critical dosage was determined by studying the rheological properties of mortars pore solutions extracted by centrifugation. Above this dosage, it can be assumed that HPG aggregates stop the water flow by plugging the porous network of a thin HPG-enriched filter cake at the interface mortar-substrate. Below this dosage, a nearly zero or hardly discernible effect of HPG on WR is obtained, which is not the case for CE.

The effect of pore solution viscosity as explanation for WR was rejected. For a given viscosity, admixtures exhibit very different WR ability. Likewise, huge differences in pore solution viscosity allow obtaining similar WR. Clearly, the key parameter to determine the dosage of WR agent in dry-mix mortar is not the induced viscosity of pore solution but the formation of polymer agglomerates. The results highlight moreover the positive impact of additional alkyl chain on WR. By enhancing overlapping, the additional substitution of alkyl chains improves the effectiveness of WR agent at low dosage.

\section{Acknowledgments}

The authors would like to acknowledge Lamberti S.p.A. for the provided products. 


\section{References}

[1] Y. Chen, X. Wu, X. Miao, J. Luo, B. Jiang, Determination of the degree of substitution of hydroxypropyl guar gum at C-6 by Pyrolysis-Gas Chromatography spectrometry, Carbohyd. Polym. 82 (3) (2010) 829-832.

[2] L. Bertrand, S. Maximilien, R. Guyonnet, Wedge Splitting Test: A test to measure the polysaccharide influence on adhesion of mortar on its substrate, Proceedings of the 11th International Congress on Polymers in Concrete, Berlin, Germany (2004) 569-576.

[3] A. Jenni, L. Holzer, R. Zurbriggen, M. Herwegh, Influence of polymers on microstructure and adhesive strength of cementitious tile adhesive mortars, Cem. Concr. Res. 35 (1) (2005) 35-50.

[4] J. Pourchez, B. Ruot, J. Debayle, E. Pourchez, P. Grosseau, Some aspects of cellulose ethers influence on water transport and porous structure of cement-based materials, Cem. Concr. Res. 40 (2) (2010) 242-252.

[5] L. Patural, P. Marchal, A. Govin, P. Grosseau, B. Ruot, O. Devès, Cellulose ethers influence on water retention and consistency in cement-based mortars, Cem. Concr. Res. 41(1) (2011) 46-55.

[6] J. Desbrieres, Cement cake properties in static filtration. Influence of polymeric additives on cement filter cake permeability, Cem. Concr. Res. 23 (2) (1993) 347-358

[7] C. Brumaud, Origines microscopiques des conséquences rhéologiques de l'ajout d'éthers de cellulose dans une suspension cimentaire, PhD Thesis, Université Paris-Est, France (2011) $161 \mathrm{pp}$.

[8] C.B. Skaggs, W.G. Rakitsy, S.F. Whitaker, Applications of rheological modifiers and superplasticizers in cementitious systems, ACI Publications SP-148 (1994) 191-207. 
[9] M. U. K. Afridi, Y. Ohama, M. Z. Iqbal, K. Demura, Water retention and adhesion of powdered and aqueous polymer-modified mortars, Cem. Concr. Comp. 17 (2) (1995) 113 118.

[10] Y. Ohama, Polymer-based admixtures, Cem. Concr. Comp. 20 (2-3) (1998) 189-212.

[11] C.-M. Capener, Properties of modern rendering systems based on mineral binders, PhD Thesis, Chalmers University of Technology of Goteborg, Sweden (2007) 168 pp.

[12] L. Patural, P. Porion, H. Van Damme, A. Govin, P. Grosseau, B. Ruot, O. Devès, A pulsed field gradient and NMR imaging investigations of the water retention mechanism by cellulose ethers in mortars, Cem. Concr. Res. 40 (9) (2010) 1378-1385.

[13] L. Patural, J.-P. Korb, A. Govin, P. Grosseau, B. Ruot, O. Devès, Nuclear magnetic relaxation dispersion investigations of water retention mechanism by cellulose ethers in mortars, Cem. Concr. Res. 42 (10) (2012) 1371-1378.

[14] C. Marliere, E. Mabrouk, M. Lamblet, P. Coussot, How water retention in porous media with cellulose ethers works, Cem. Concr. Res. 42 (11) (2012) 1501-1512.

[15] K. M. Green, M. A. Carter, W. D. Hoff, M. A. Wilson, The effects of lime and admixtures on the water-retaining properties of cement mortars, Cem. Concr. Res. 29 (11) (1999) $1743-1747$.

[16] M. Carter, K. Green, M. Wilson, W. Hoff, Measurement of the water retentivity of cement mortars, Adv. Cem. Res. 15 (4) (2003) 155-159.

[17] R. Hendrickx, S. Roels, K. Van Balen, Measuring the water capacity and transfer properties of fresh mortar, Cem. Concr. Res. 40 (12) (2010) 1650-1655.

[18] E. Knapen, D. Van Gemert, Cement hydration and microstructure formation in the presence of water-soluble polymers, Cem. Concr. Res. 39 (1) (2009) 6-13. 
[19] D. A. Silva, V. M. John, J. L. D. Ribeiro, H. R. Roman, Pore size distribution of hydrated cement pastes modified with polymers, Cem. Concr. Res. 31 (8) (2001) 1177-1184.

[20] D. Bülichen, J. Kainz, J. Plank, Working mechanism of methyl hydroxyethyl cellulose (MHEC) as water retention agent, Cem. Concr. Res. 42 (7) (2012) 953-959.

[21] D. Bülichen, J. Plank, Role of colloidal polymer associates for the effectiveness of hydroxyethyl cellulose as a fluid loss control additive in oil well cement, J. Appl. Polym. Sci. 126 (S1) (2012) E25-E34.

[22] D. Bülichen, J. Plank, Mechanistic study on carboxymethyl hydroxyethyl cellulose as fluid loss control additive in oil well cement, J. Appl. Polym. Sci. 124 (3) (2012) 2340-2347. [23] A. Jenni, R. Zurbriggen, L. Holzer, M. Herwegh, Changes in microstructures and physical properties of polymer-modified mortars during wet storage, Cem. Concr. Res. 36 (1) (2006) 79-90.

[24] A. De Gasparo, M. Herwegh, R. Zurbriggen, K. Scrivener, Quantitative distribution patterns of additives in self-leveling flooring compounds (underlayments) as function of application, formulation and climatic conditions, Cem. Concr. Res. 39 (4) (2009) 313-323. [25] J. Plank, Applications of biopolymers and other biotechnological products in building materials, Appl. Microbiol. Biotechnol. 66 (1) (2004) 1-9.

[26] M. Cappellari, A. Daubresse, M. Chaouche, Influence of organic thickening admixtures on the rheological properties of mortars: Relationship with water-retention, Constr. Build. Mater. 38 (0) (2013) 950-961.

[27] T. Poinot, A. Govin, P. Grosseau, Impact of hydroxypropylguars on the early age hydration of Portland cement, Cem. Concr. Res. 44 (0) (2013) 69-76. 
[28] M. Lasheras-Zubiate, I. Navarro-Blasco, J. M. Fernández, J. I. Álvarez, Effect of the addition of chitosan ethers on the fresh state properties of cement mortars, Cem. Concr. Comp. 34 (8) (2012) 964-973.

[29] B. Biasotti, M. Giudici, V. Langella, U. Pfeiffer, Highly substituted hydroxypropylguar: a strong contribution to construction chemistry, in:Proceedings of the Third International Drymix Mortar Conference, Nürnberg, Germany (2011) Ed. Ferdinand Leopolder, ISBN: 978-3-9814004-1-0.

[30] D. Risica, M. Dentini, V. Crescenzi, Guar gum methyl ethers. Part I. Synthesis and macromolecular characterization, Polymer 46 (26) (2005) 12247-12255.

[31] EN 196-1, Methods of testing cement — Part 1: Determination of strength (2006).

[32] Standard C1506-09. Standard test Method fo Water Retention of Hydraulic CementBased Mortars and Plasters. Am Soc Test Mat 2009.

[33] NF DTU 26.1, Travaux d'enduits de mortiers (2008).

[34] W. W. Graessley, The entanglement concept in polymer rheology, Ed. Springer, Berlin Heidelberg (1974) 1-179.

[35] E. R. Morris, A. N. Cutler, S. B. Ross-Murphy, D. A. Rees, J. Price, Concentration and shear rate dependence of viscosity in random coil polysaccharide solutions, Carbohyd. Polym. 1 (1) (1981) 5-21.

[36] C. Clasen, W. M. Kulicke, Determination of viscoelastic and rheo-optical material functions of water-soluble cellulose derivatives, Prog. Polym. Sci. 26 (9) (2001) 1839-1919.

[37] G. Robinson, S. B. Ross-Murphy, E. R. Morris, Viscosity-molecular weight relationships, intrinsic chain flexibility, and dynamic solution properties of guar galactomannan, Carbohyd. Res. 107 (1) (1982) 17-32. 
[38] R. Lapasin, L. De Lorenzi, S. Pricl, G. Torriano, Flow properties of hydroxypropyl guar gum and its long-chain hydrophobic derivatives, Carbohyd. Polym. 28 (3) (1995) 195202.

[39] M. Oblonšek, S. Šostar-Turk, R. Lapasin, Rheological studies of concentrated guar gum, Rheol. Acta 42 (2003) 491-499.

[40] W. Sittikijyothin, D. Torres, M. P. Gonçalves, Modelling the rheological behaviour of galactomannan aqueous solutions, Carbohyd. Polym. 59 (3) (2005) 339-350.

[41] D. Risica, A. Barbetta, L. Vischetti, C. Cametti, M. Dentini, Rheological properties of guar and its methyl, hydroxypropyl and hydroxypropyl-methyl derivatives in semidilute and concentrated aqueous solutions, Polymer 51 (9) (2010) 1972-1982.

[42] W. M. Kulicke, A. H. Kull, W. Kull, H. Thielking, J. Engelhardt, J. B. Pannek, Characterization of aqueous carboxymethylcellulose solutions in terms of their molecular structure and its influence on rheological behaviour, Polymer 37 (13) (1996) 2723-2731. [43] J. Pourchez, P. Grosseau, B. Ruot, Changes in C3S hydration in the presence of cellulose ethers, Cem. Concr. Res. 40 (2) (2010) 179-188.

[44] S. Simon, J. Dugast, D. Le Cerf, L. Picton, G. Muller, Amphiphilic polysaccharides. Evidence for a competition between intra and intermolecular associations in dilute system, Polymer 44 (26) (2003) 7917-7924.

[45] Y. Cheng, K. M. Brown, R. K. Prud'homme, Characterization and Intermolecular Interactions of Hydroxypropyl Guar Solutions, Biomacromolecules 3 (3) (2002) 456-461. 